Gazi University
Journal of Science
http://dergipark.gov.tr/gujs

\title{
Drug Delivery Application of Poly (2-hydroxyethyl methacrylate)/Ethylene glycol Dimethacrylate Composite Hydrogel
}

\author{
Nihal ERMIS $1,2, *$ (iD \\ ${ }^{I}$ Samsun University, Faculty of Engineering, Department of Fundamental Sciences, 55420 Ondokuzmayls, Samsun, Turkey \\ ${ }^{2}$ Samsun University, Faculty of Engineering, Department of Biomedical Engineering, 55420 Ondokuzmayls, Samsun, Turkey

\section{Highlights} \\ - This paper focuses on utilization of a p(HEMA) hydrogel system for drug delivery application. \\ - A composite $\mathrm{p}(\mathrm{HEMA})$ hydrogel was synthesized via free radical polymerization at room temperature. \\ - FTIR, TGA, SEM images, swelling behavior were investigated for the characterization of the samples. \\ - The release kinetics were also investigated to exhibit the delivery potential of the composite hydrogel.
}

Article Info

Received: 04 June 2021

Accepted: 20 Dec 2021

Keywords

Hydrogel

PHEMA

Amoxicillin

Drug Delivery

Polymerization

\begin{abstract}
Hydrogels have become advantageous materials used in biomedical applications as catheter, soft contact lenses or drug carrying vehicle due to their advantageous features like biocompatibility, insoluble property in aqueous solutions, their resistance to environmental conditions like $\mathrm{pH}$, temperature. Periodontitis is an inflammatory disease which leads to the loss of tissue attachment, gingival recession, tooth loss or periodontal pocket formation. In this study, it was aimed to develop antibiotic loaded hydrogels composed of HEMA and EGDMA for the possible treatment of periodontitis. The in vitro drug delivery characteristics were determined with amoxicillin, which was selected as a proper antibiotic sample for the treatment of periodontitis. In conclusion, the $\mathrm{H} 1$ named hydrogel sample was proven to have a possible performance as an amoxicillin reservoir.
\end{abstract}

\section{INTRODUCTION}

The drug delivery technology is a fast-developing area which is useful for decreasing side effects of pharmaceuticals and increasing drug efficacy. The drug delivery technology offers many advantages such as targeting the release to a specific address and extending the desired effect time of the drug. Attaching a drug to a polymer provides drug safety and increases the desired effect of the drug [1]. Different variations of chemical functionalization like grafting techniques or controlled radical polymerization (CRP) methods have provided new opportunities to drug-delivery science via preparation of smart and functional delivery matrices [2]. Hydrogels are functional and promising delivery reservoirs due to their high-water content, elastic features like natural tissues, and their permeability to body fluids. They are three-dimensional, waterswollen hydrophilic polymer networks produced by the simple reaction of one or more monomers. They exhibit the ability to retain a significant amount of water within its structure. They can maintain their integrity in the presence of large amount of water molecules, through chemical or physical crosslinks [3]. Hydrogels have many more advantages than other biomaterials due to their increased biocompatibility, adjustable biodegradability, porous structure, and desired mechanical strength [4]. All these advantages, especially the high-water content provides hydrogels strong biocompatibility. They are promising biomaterials with great potential for biomedical applications [5]. Due to all these features, hydrogels have a wide range of application including tissue engineering [6,7], self-healing materials [8,9], drug delivery $[10,11]$, biosensors $[12,13]$ and for dental implant to provide dental hygiene or dental treatment [14-16]. 
The poly (2-hydroxyethyl methacrylate) (p(HEMA)) hydrogel is one of the most used polymeric materials in materials science. The p(HEMA) hydrogels have excellent biocompatibility, high thermal stability, resistance to high acidic and alkali medium and adjustable mechanical features. They have physical properties like living tissue and the hydroxyl groups on their surface provide attachment of different types of molecules to interact with hydrogels [17]. Thus, hydrogels have become advantageous materials used in biomedical applications as catheter, soft contact lenses or drug carrying vehicle [18].

Amoxicillin (AMX) is one of the most used antibiotics for medical therapy. The main aim of using amoxicillin is treating or preventing a wide range of bacterial infections such as lower respiratory tract, ear, throat, and dental infections both for humans and animals [19]. During years researchers have discovered that amoxicillin is found in ground water, drinking water, meat, or food of animal origin due to excess utilization of amoxicillin [20-22]. The unconscious utilization of amoxicillin for both human health and in food industry leads the accumulation of the antibiotic in food products such as, meat, milk, eggs and in environment samples from ground water to drinking water. However, adequate utilization of amoxicillin is crucial for both human health and environment. Therefore, the controlled drug delivery is a keystone for the solution of this problem and for an adequate treatment.

Periodontitis is an inflammatory disease which affects $10-15 \%$ of the world population approximately. It causes periodontal pocket formation, gingival recession, and the loss of tissue attachment. Due to the formation of bacterial plaque and the respective host immune response inflammation initiates in the gingival area [23]. The inflammation starts with the release of pro-inflammatory cytokines and hence the activation of the immune system. The lymphocytes and phagocytes recruit to the inflamed area and the response of the immune system induce inflammation [24]. Dental health plays an important role in the whole organism. Because stroke, diabetes, atherosclerosis, coronary heart disease is also associated with the periodontitis. [25]. Therefore, it is important to maintain oral health and avoid smoking and provide proper dental cleaning. In the case of progressed periodontitis, mechanical treatment is disadvantageous in terms of time consumption, difficulty to use instruments in the inflamed area and the chemical treatment is disadvantageous in terms of the presence of multiple drug-resistant microorganism, adverse drug reactions and the inability to achieve high gingival crevicular fluid concentration [26].

With the development of science, the disadvantages of these conventional treatments are coped with the different types of drug delivery systems. The main aim of the controlled drug delivery is to deliver drugs to the target sites at desired amount and time. Thus, the drug efficacy and pharmacokinetics improves while side effects minimize [27]. A drug-loaded polymeric system, e.g., a hydrogel, is designed to release the drug from the bulk material. Through swelling-controlled system provided by water penetration, a hydrogel can deliver drug into the targeted area while retaining the therapeutic effect for a long term. The rate is controlled by the penetration of water into the hydrogel system. A dry hydrogel interacts with water or body fluids when they placed in the body and the hydrogel absorbs water and swells. Through swelling, mesh size of the hydrogel increases which led the drug to be released to the external environment over the swollen network [28]. A local drug delivery system through hydrogel is advantageous in terms of convenience in utilization, direct access, enhanced efficacy, and improvement of patient compliance [23]. Besides, the utilization of any topical cream or ointment for periodontitis would be less effective due to continuous saliva production. Also, previous studies have showed that hydrogels have great potential in dental therapy against periodontitis due to easy adherence to dental pocket and their semi-solid nature $[23,29]$.

The current study is a demonstration on the application of antibiotic loaded hydrogel-based drug delivery system to treat periodontitis as an implant. The p(HEMA) hydrogel has advantages such as a suitable structure as a drug carrier, biodegradability, high biocompatibility, and high specific surface area. The drug release profile of $\mathrm{p}$ (HEMA) hydrogel has been investigated by using amoxicillin as the model drug. The structure, swelling characteristics and drug release performance of the amoxicillin loaded p(HEMA) hydrogel were evaluated in the study. For optimization and investigation of the cross-linker amount, the hydrogels were synthesized with different amounts of cross-linker. Under the optimum conditions, the obtained hydrogel has proved that it could rapidly and continuously release antibiotic to maintain the drug concentration in a longer duration. 


\section{MATERIALS AND METHODS}

\subsection{Materials}

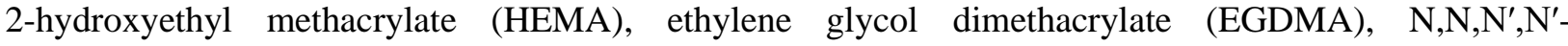
tetramethylethylenediamine (TEMED) and ammonium persulfate (APS) were purchased from SigmaAldrich (U.S.A). All other chemicals were obtained from Merck (Darmstadt, Germany). The chemicals used in the experiments were of analytical grade.

\subsection{Preparation of Hydrogels}

In this study, hydrogels were synthesized by crosslinking of HEMA (used as monomer) in the presence of EGDMA. The polymerization procedure can be seen on Figure 1. Briefly, aqueous solution of HEMA including EGDMA was prepared according to amounts listed in Table 1. The pre-polymerization solution was stirred under a nitrogen atmosphere for $30 \mathrm{~min}$. After that, $20 \mu \mathrm{L}$ of TEMED and $10 \mathrm{mg}$ of APS were added to initiate the polymerization. The solution was stirred continuously for 10 min under $\mathrm{N}_{2}$ atmosphere. Then the solution was transferred into a $1.5 \mathrm{~mm}$ diameter, $3 \mathrm{~cm}$ high, screw-capped glass tubes and sealed immediately. Then it was left at room temperature for overnight to complete the polymerization process.

Table 1. Contents of synthesized hydrogels

\begin{tabular}{|l|l|l|l|l|}
\hline Nomenclature & HEMA $(\mathrm{mL})$ & EGDMA $(\mathrm{mL})$ & APS $(\mathrm{mg})$ & TEMED $(\mu \mathrm{L})$ \\
\hline H1 & 0.5 & 0.005 & 10 & 20 \\
\hline H2 & 0.5 & 0.01 & 10 & 20 \\
\hline H3 & 0.5 & 0.025 & 10 & 20 \\
\hline
\end{tabular}

After polymerization, the hydrogel samples were cut into equal size of discs and moved into deionized water to completely remove the unreacted reagents. The samples were kept in deionized water for 3 days with refreshing the water several times every day. Then hydrogels were kept in the oven at $35{ }^{\circ} \mathrm{C}$ until the hydrogels reached constant weight and stored in desiccator.

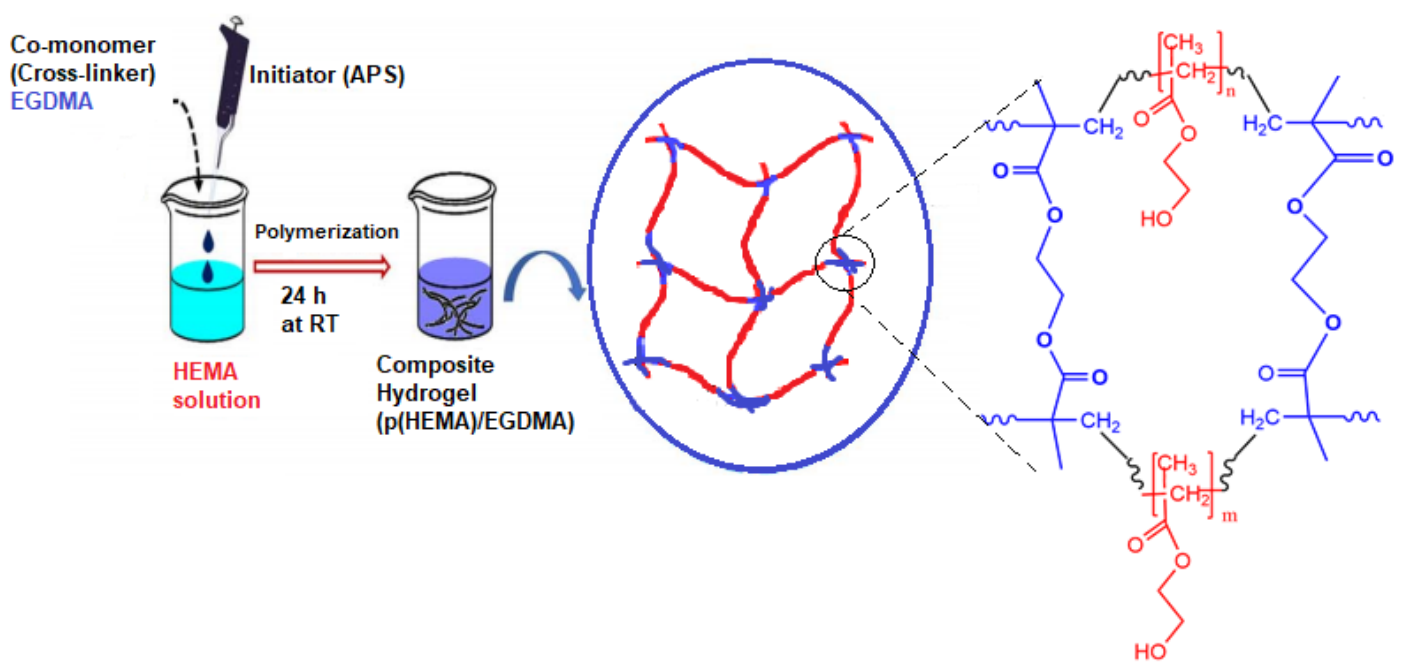

Figure 1. Preparation of poly (HEMA-co-EGDMA) hydrogel 


\subsection{Characterization}

\subsubsection{Fourier transform infrared (FTIR) spectroscopy}

FTIR spectra of hydrogels were obtained via a Nicolet iS10 FTIR instrument (Thermo Fisher Scientific, USA). Nitrogen gas was passed through the sample chamber for 10 minutes to remove the moisture and carbon dioxide of the air. The hydrogel sample was placed in the sample holder of the device and spectrum was taken in the wave number range of $400-4000 \mathrm{~cm}^{-1}$ by measuring the total amount of reflection on the surface.

\subsubsection{Thermo gravimetric (TGA/DTA/DSC) analysis}

Thermal behavior of poly (HEMA-co-EGDMA) hydrogels was analyzed with thermogravimetric analyzer (TGA / DTA 6300, SII EXSTAR 6000, Seiko Instruments Inc., USA) and differential scanning calorimetry (DSC). TGA analysis was performed in the temperature range of $25-600{ }^{\circ} \mathrm{C}$, at a heating rate of $10^{\circ} \mathrm{C} / \mathrm{min}$. DSC measurements were performed by using DSC-60 Shimadzu differential scanning calorimeter to obtain the effect of cross-linker amount on glass transition temperature of the hydrogel samples. Both TGA and DSC measurements were performed under nitrogen atmosphere.

\subsubsection{Scanning electron microscopy (SEM)}

The surface morphology of the hydrogel samples was investigated via SEM images. The sample was sputter-coated with gold and palladium. SEM images were taken by accelerating the electrons with $15 \mathrm{kV}$ with the JEOL JSM-7001F (JEOL USA, Inc.) brand device.

\subsubsection{Swelling characteristics}

For characterization of swelling behavior of the hydrogel samples, they were cut into a diameter of $10 \mathrm{~mm}$ and a height of $3 \mathrm{~mm}(\mathrm{n}=3)$ to prepare disk-shaped hydrogel samples. After drying hydrogel discs to constant weight, they were immersed in deionized water at $37^{\circ} \mathrm{C}$ and weighed at certain time intervals after removal of the excess water on the surface. The equilibrium water content of the hydrogels was investigated through swelling and drying the sample consecutively $(n=3)$. The water uptake (WU) and equilibrium water content (EWC) of hydrogel was calculated using Equation (1) and (2), respectively

$$
\begin{aligned}
& W U=\left(W_{s}-W_{d}\right) / W_{d}, \\
& E W C=\frac{\mathrm{W}_{e q}-\mathrm{W}_{0}}{W_{0}} x 100 .
\end{aligned}
$$

In Equation (1), $\mathrm{W}_{\mathrm{s}}$ stands for the weight of swollen sample at a t time and $\mathrm{W}_{\mathrm{d}}$ stands for the weight of the dry sample. In Equation (2), $\mathrm{W}_{\text {eq }}$ stands for the weight of swollen sample at equilibrium and $\mathrm{W}_{0}$ stands for the weight of the dry sample.

\subsubsection{Drug loading}

The in vitro drug delivery performance of the hydrogel samples was performed through release of amoxicillin from the poly (HEMA-co-EGDMA) hydrogel discs. The weighed and dried hydrogel discs were immersed in $10 \mu \mathrm{M}$ amoxicillin solution $(20 \mathrm{~mL}$, in $\mathrm{pH} 7.4)$ until equilibrium to load drug. After loading, the samples were washed with distilled water and dried until a constant weight in the drying oven. The loading amount of the drug was calculated via the weight method by using Equation (3):

$$
\text { Amount of loaded drug }=\mathrm{W}_{0}-\mathrm{W}_{1}
$$


where $\mathrm{W}_{0}$ is the weight of drug loaded hydrogel and $\mathrm{W}_{1}$ is the weight of the unloaded hydrogel sample [30].

\subsubsection{Drug release studies}

In vitro drug release studies were performed in $\mathrm{pH} 7.4$ phosphate buffer solution, which is physiological $\mathrm{pH}$ and the $\mathrm{pH}$ in the oral cavity maintained by saliva to exhibit the eligibility of drug release from the hydrogel disc in oral environment [31]. The weighed, amoxicillin loaded, and dried hydrogel discs were transferred in buffer solution at $37^{\circ} \mathrm{C}$. During the study, solution was stirred at a rotating speed of $150 \mathrm{rpm}$. Aliquots of $2 \mathrm{~mL}$ of the samples were removed and a fresh medium of the same volume was added to maintain sink condition. The collected samples were analyzed by using a UV-vis-spectrophotometer (Thermo Fisher Scientific Inc., Thermo Insight 2).

\subsubsection{Kinetics of drug release}

The mechanism and rate of amoxicillin release from the hydrogels were investigated via releasing data and equations for zero order and first order release, Higuchi and Korsemeyer-Peppa's kinetic release models to perform the in vitro drug release profile. For zero order release Equation (4) is used as follows:

$$
Q=K_{0} t .
$$

Here $\mathrm{Q}$ stands for the amount of the drug released at time $\mathrm{t}$ and $\mathrm{K}_{0}$ is the zero order release rate constant. Equation (5) is used as follows for the first order release

$$
\log (100-\mathrm{Q})=\log 100-\mathrm{K}_{1} \mathrm{t} .
$$

Here, $K_{1}$ is the first order release rate constant. The releasing data was fitted to the Equation (6) and (7) to find Higuchi model kinetic release and Korsemeyer-Peppa model kinetic release, which are usually utilized to describe the drug release behavior from polymeric systems [32]

$$
\begin{aligned}
& Q=K_{2} \sqrt{t} \\
& \log \left(\frac{M t}{M \infty}\right)=\log K+n \log t
\end{aligned}
$$

Here, $\mathrm{K}_{2}$ is the Higuchi model release rate constant; $\mathrm{M}_{\mathrm{t}}$ and $\mathrm{M}_{\infty}$ refer to the amount of released drug at time $\mathrm{t}$ and after infinite time, respectively. $\mathrm{n}$ is the diffusion exponent indicative of the mechanism of drug release. If the exponent value (n) is smaller than 0.5, then the drug release mechanism based on quasifickian diffusion. If $\mathrm{n}$ is equal to 0.5 then release mechanism is fickian diffusion and if the value is between 0.5 and 1 , then it is anomalous diffusion coupled with erosion. If exponent value is 1 , then the release mechanism is indicative of Case-II Transport or typical zero order. If it is bigger than 1, than the release mechanism fits to non-fickian super Case II and the diffusion exponent was based on Korsmeyer-Peppas equation.

\section{THE RESEARCH FINDINGS AND DISCUSSION}

\subsection{Preparation of Hydrogels}

The HEMA-based hydrogels are advantageous because of their mechanical strength, and they are not absorbed by the body due to their mechanical strength. Besides they are easy and convenient to produce and for biomedical utilization. HEMA is a hydrophilic monomer which contains hydroxyl groups and EGDMA is the cross-linker which is generally used for p(HEMA) synthesis [33]. p(HEMA-co-EGDMA) hydrogels were synthesized with increasing amounts of cross linker as mentioned in Table 1 and the polymerization is illustrated in Figure 1. Hydrogel samples were polymerized via free-radical 
polymerization. After adding TEMED to pre-polymerization solution, it reacted with APS and sulfate free radical was produced due to splitting of the persulfate ions. The formed free radicals initiated the polymerization through crosslinking, where amount of the cross-linker defines binding density. The greater the amount of cross linker in the polymer, polymeric chains hold more tightly and the gaps between them are reduced. This situation also affects the swelling rate of the polymer, i.e., the amount of cross linker in the polymeric structure the higher, the rate of swelling of the polymer decreases.

\subsection{Characterization}

\subsubsection{Fourier transform infrared (FTIR) spectroscopy}

The components of the synthesized $\mathrm{p}$ (HEMA-co-EGDMA) were examined by FTIR spectroscopy (Figure 2). On the spectra it can be clearly seen that increasing amount of EGDMA incorporated properly into the structure as abbreviated from $\mathrm{H} 1$ to $\mathrm{H} 3$. All characteristic peaks could be seen on the spectra. In the spectra, the broad peak at $3450 \mathrm{~cm}^{-1}$ was attributed to $-\mathrm{OH}$ stretching vibration. However, in the literature HEMA monomer has a broad and strong peak of $-\mathrm{OH}$ in FTIR spectrum, which is observed in these spectra narrower, which exhibits the bonding between HEMA and EGDMA [34]. Besides, the peak at $1730 \mathrm{~cm}^{-1}$ represented $\mathrm{C}=\mathrm{O}$ stretching vibration of ester groups in EGDMA. The bands near $1600 \mathrm{~cm}^{-1}$ assigned to the $\mathrm{C}=\mathrm{C}$ bond stretching vibrations and the band strengths with the increasing amount of cross linker, which proves that the polymerization takes place [35]. The peak at $2950 \mathrm{~cm}^{-1}$ and $1118 \mathrm{~cm}^{-1}$ were assigned to the aliphatic stretching vibration of $-\mathrm{CH}_{2}-,-\mathrm{CH}$ and $-\mathrm{CH}_{3}$ groups, respectively $[36,37]$.

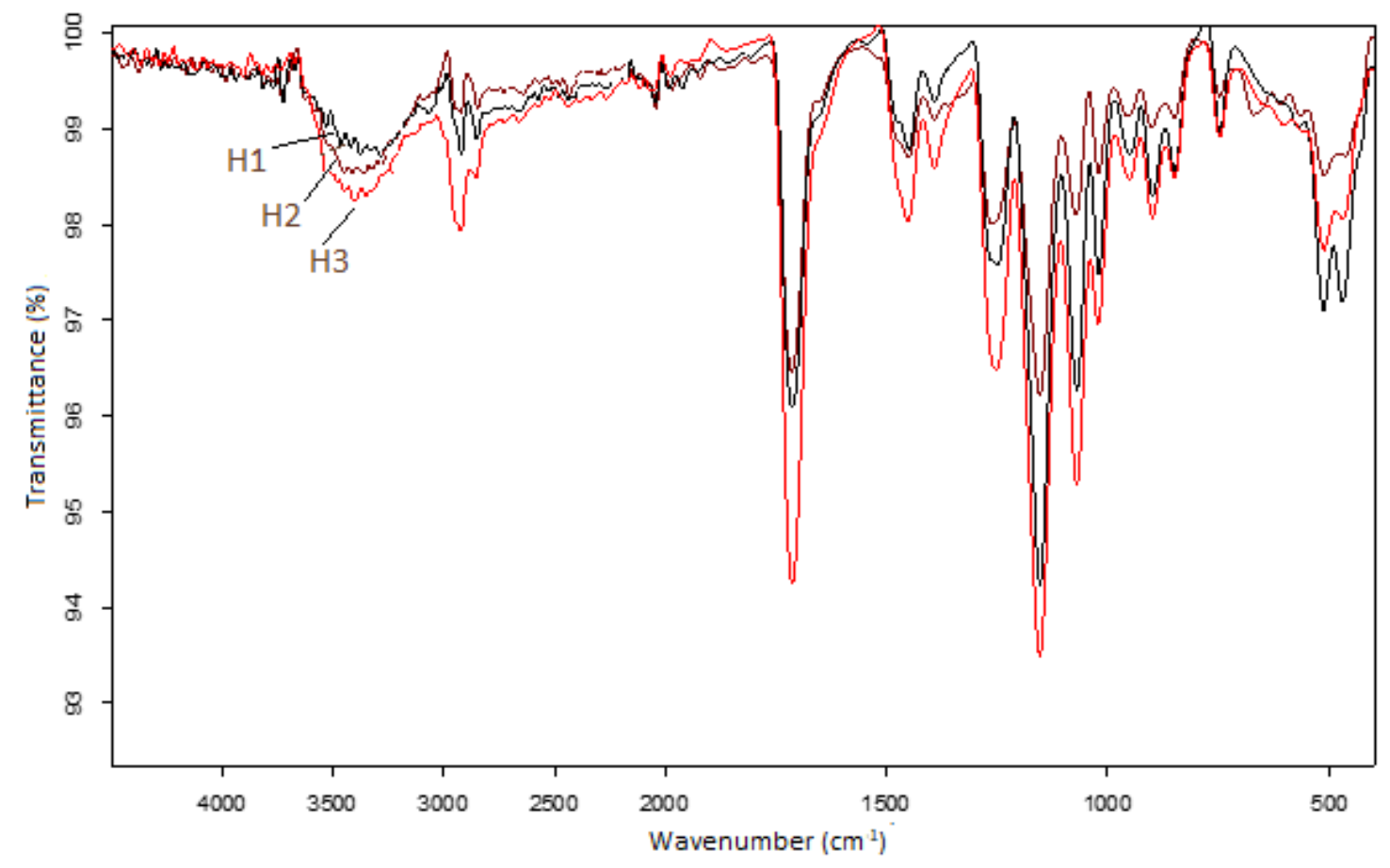

Figure 2. FTIR spectra of the hydrogel samples

\subsubsection{Thermo gravimetric (TGA/DTA/DSC) analysis}

In thermal gravimetric analyses of hydrogel samples, no significant mass loss was observed till the onset of thermal degradation of the hydrogel samples (Figure 3). It clearly indicates that all components of the polymers have constituted structure of the hydrogels without remaining any of the monomer. The increase of cross linker concentration provides an increase of degradation temperature. The values were $230{ }^{\circ} \mathrm{C}, 285$ ${ }^{\circ} \mathrm{C}$ and $295{ }^{\circ} \mathrm{C}$ for networks containing 1 vol\% $(\mathrm{H} 1), 2$ vol\% $(\mathrm{H} 2)$ and 5 vol\% (H3) cross linker, respectively. The amount of the cross linker seemed to have a positive effect on thermal stability of the hydrogel networks. In the range of $300-450{ }^{\circ} \mathrm{C}$, the weight of all hydrogel samples decreased because of 
the thermo-degradation of molecule skeleton. These results present that $\mathrm{p}$ (HEMA-co-EGDMA) hydrogels are thermo-stable at temperature lower than $200^{\circ} \mathrm{C}$ and own high hydrophilicity.

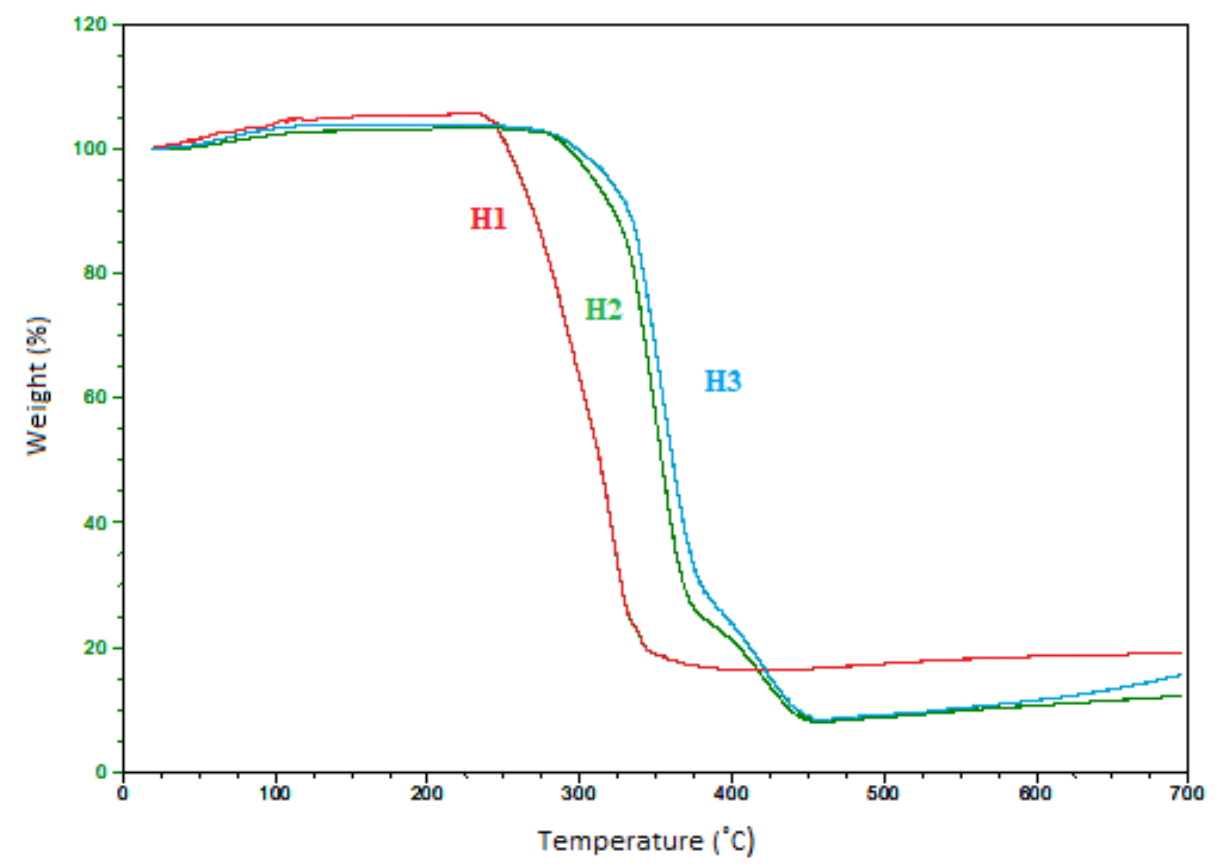

Figure 3. TGA analysis of the hydrogel samples

\subsubsection{Scanning electron microscopy (SEM)}

The surface morphology of the hydrogel samples was investigated via SEM and the images were given in Figure 4. The difference of the structure based on cross linker amount can be seen in figures. H3 contains the largest amount of cross linker and obtains more blocked structure than other samples. H1 has more porous structure and the least amount of cross linker, which exhibits the relationship between the amount of cross linker and compact structure. Besides, H1 hydrogel displays the highest swelling ratio while $\mathrm{H} 3$ displays the least swelling ratio. The result of the analyses exhibits that the swelling ratio, porousness, and compact structure of hydrogels are directly associated with each other. 


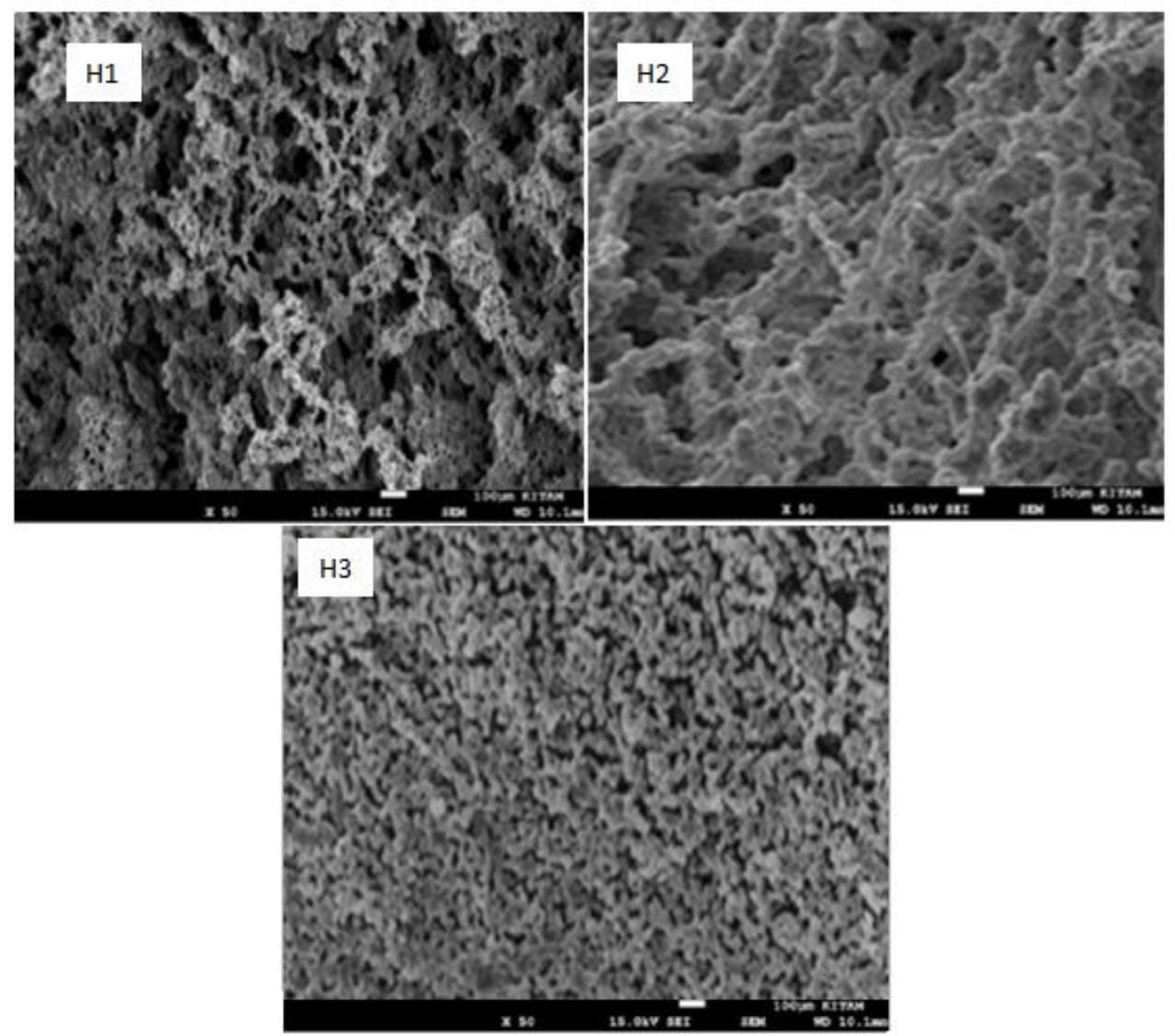

Figure 4. SEM images of the hydrogels

\subsubsection{Swelling characteristics}

The swelling kinetics of the samples was achieved by immersing them in deionized water at $37{ }^{\circ} \mathrm{C}$ and shown in Figure 5. The swelling ratio and water uptake of the samples increased until equilibrium as can be seen on the Figure 5.A and 5.B. H1 exhibited the fastest and largest swelling rate than the other samples due to its large pore size. High cross-linker amount leads polymer structure to be denser which causes a disadvantage for swelling of hydrogel as can be seen swelling ratios of $\mathrm{H} 2$ and $\mathrm{H} 3$ [38].
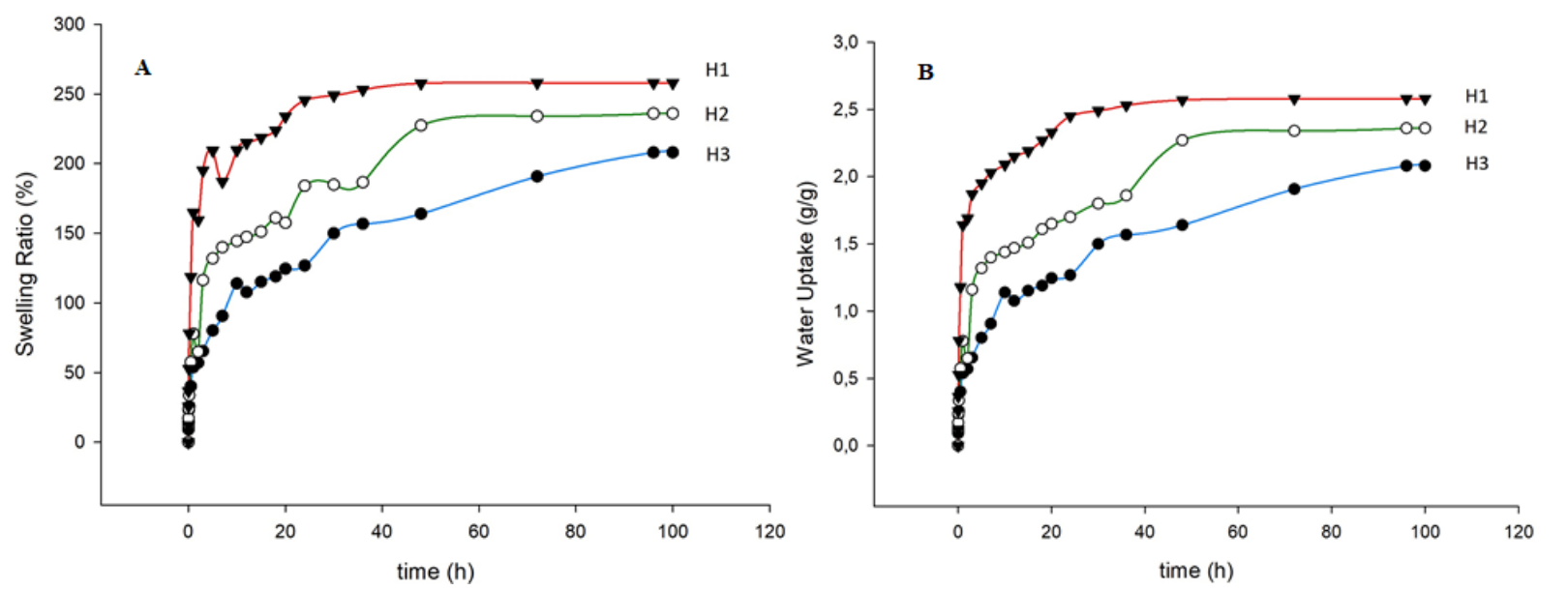

Figure 5. A. Swelling behavior and B. water uptake amounts of the hydrogel samples (H1:red, H2: green, H3:blue)

Equilibrium water content (EWC) analysis was utilized to test whether the swelling capacity of polymers is stable. EWC of the samples for the first swelling were found as $292.4 \pm 3.7,233.4 \pm 5$ and $201.2 \pm 3$ for H1, 
$\mathrm{H} 2$ and $\mathrm{H} 3$, respectively (Table 2). EWC values have indicated that swelling properties of the p(HEMA) samples were reversible and supported relation between swelling ratio and pore size.

Table 2. Equilibrium water contents of the hydrogels

\begin{tabular}{|l|l|l|l|}
\hline \multirow{2}{*}{ Hydrogel Sample } & \multicolumn{3}{|c|}{ Equilibrium Water Content (\%) } \\
\cline { 2 - 4 } & Swelling Number 1 & Swelling Number 2 & Swelling Number 3 \\
\hline H1 & $292.4 \pm 3.7$ & $296.2 \pm 2.4$ & $297 \pm 3.2$ \\
\hline H2 & $233.4 \pm 5$ & $236.7 \pm 9$ & $250.4 \pm 4.1$ \\
\hline H3 & $201.2 \pm 3$ & $191.6 \pm 4.8$ & $197 \pm 2.5$ \\
\hline
\end{tabular}

\subsubsection{Drug loading and release studies}

Each of the hydrogel disks (H1, H2 and H3), prepared with different amounts of cross linkers, were kept in $20 \mathrm{~mL}$ volume $20 \mu \mathrm{L}$ amoxicillin solutions prepared with $\mathrm{pH} 7.4$ phosphate buffer at $37^{\circ} \mathrm{C}$ for 24 hours by stirring the solutions at $150 \mathrm{rpm}$. After the end of the period, the disc was washed with phosphate buffer. For release study hydrogel disc was placed in $20 \mathrm{~mL}$ phosphate buffer, and amoxicillin release was continued for 72 hours. At the specified time intervals, $2 \mathrm{~mL}$ of sample was taken from the buffer medium and $2 \mathrm{~mL}$ of phosphate buffer was replaced to maintain the sink condition. The sink condition is provided to prevent the uneven increase in the concentration of the active substance in the environment. Under sink condition, the dissolved active substance concentration $\left(\mathrm{C}_{\mathrm{t}}\right)$ at any time $\mathrm{t}$ should not exceed $20 \%$ of the solubility $\left(\mathrm{C}_{\mathrm{s}}\right)$ of this active substance in the medium. $\left(\mathrm{C}_{\mathrm{t}}<0.2 \mathrm{Cs}\right)$ [39].

All the hydrogel samples exhibited burst release in the first 2 hours (Figure 6). The reason of this burst release was easy diffusion of the drug molecules, which was near to hydrogel surface, to the release medium. In the next 24 hours, while the release from H1 hydrogel continued to increase, the release from $\mathrm{H} 2$ and $\mathrm{H} 3$ hydrogels was relatively less than $\mathrm{H} 1$. 


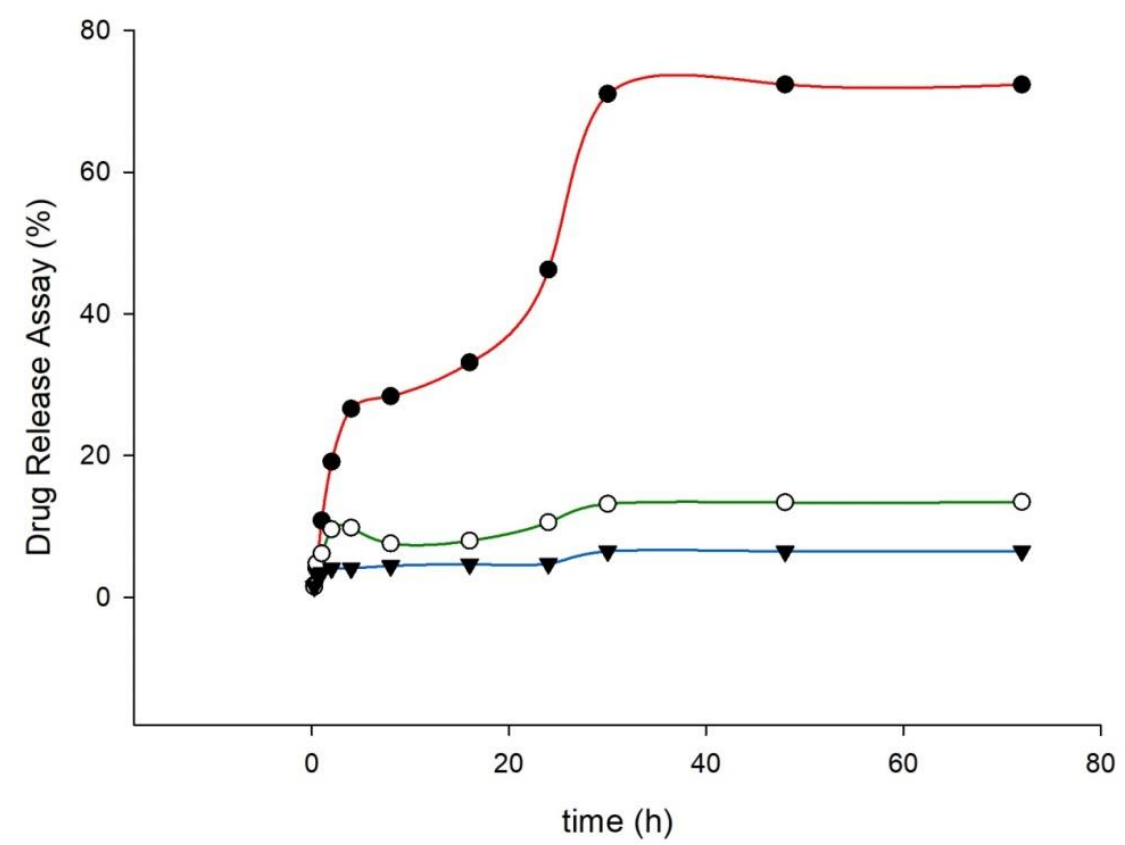

Figure 6. Drug release assay of the hydrogel samples (H1: red, H2: green, H3:blue)

The release mechanism in the hydrogel system is generally diffusion controlled and amoxicillin release from hydrogels was as follows; First, amoxicillin loaded hydrogels interacted with the phosphate buffer medium and the medium liquid penetrated to the hydrogel and hydrogel structure began to swell. At the same time, amoxicillin on the hydrogel began to be released. Later, as the polymer matrix absorbed the ambient fluid, amoxicillin began to diffuse from the matrix and its release into the environment continued increasingly for a certain period. In order to determine the concentration of the release solution, samples were taken from the $\mathrm{pH} 7.4$ phosphate buffer at regular intervals and analyzed with UV-Vis spectrophotometer (Thermo Fisher Scientific Inc., Thermo Insight 2). The release studies were continued for 72 hours. However, it was observed that after 30 hours the release rate came to a more stable value. Besides, the release rate of amoxicillin from $\mathrm{H} 1$ hydrogel was faster than $\mathrm{H} 2$ and $\mathrm{H} 3$ hydrogel showed the lowest release rate and amount. This release profile was like the swelling ratio discussed above and was compatible with literature data [40]. As can be seen from the release results, with the increase of cross linker amount in the polymeric structure, the hydrophobicity increased, pore size decreased, and the diffusion of the solution became more difficult. Consequently, the swelling rate and release profile decreased as expected.

\subsubsection{Kinetics of drug release}

The data, obtained through amoxicillin release from the hydrogels, was fitted to drug release kinetics models, which was mentioned before. The $n, k$ and regression coefficients $\left(\mathrm{R}^{2}\right)$ values are presented in Table 3 and the best-fit model was selected based on $\mathrm{R}^{2}$. It is observed that kinetics of amoxicillin release fits better to the Higuchi model with $\mathrm{H} 1$ hydrogel. H1 fits better for controlled release of amoxicillin with a $\mathrm{R}^{2}$ value of 0.99 . Besides, the release exponent (n) value indicates the mechanisms to describe how the active compound released from their matrix. In this case, the $n$ value of 0.54 indicates the process of polymeric chain relaxation is much greater than solvent diffusion, which exhibits the swelling behavior is responsible of drug release from the hydrogel [41]. Besides, the diffusion exponential value (k) has decreased with the increase of the cross-linker amount, which confirms that the drug release is based on swelling. 
Table 3. Release kinetics of amoxicillin from the prepared hydrogels

\begin{tabular}{|l|l|l|l|l|l|l|l|l|l|}
\hline \multirow{2}{*}{$\begin{array}{l}\text { Hydrogel } \\
\text { Sample }\end{array}$} & \multicolumn{2}{l|}{ Zero Order } & \multicolumn{2}{l|}{ First Order } & \multicolumn{2}{l|}{ Higuchi Model } & \multicolumn{2}{l|}{ Korsemeyer-Peppa's Model } \\
\cline { 2 - 11 } & $\mathrm{R}^{2}$ & $\mathrm{~K}_{0}$ & $\mathrm{R}^{2}$ & $\mathrm{~K}_{1}$ & $\mathrm{R}^{2}$ & $\mathrm{~K}$ & $\mathrm{R}^{2}$ & $\mathrm{n}$ value & $\mathrm{K}$ \\
\hline $\mathrm{H} 1$ & 0.93 & 3.03 & 0.89 & -0.0029 & 0.99 & 11.67 & 0.93 & 0.54 & 0.57 \\
\hline $\mathrm{H} 2$ & 0.92 & 0.22 & 0.79 & -0.0002 & 0.69 & 1.37 & 0.69 & 0.29 & 0.43 \\
\hline $\mathrm{H} 3$ & 0.83 & 0.17 & 0.73 & -0.0001 & 0.59 & 0.59 & 0.79 & 0.2 & 0.04 \\
\hline
\end{tabular}

\section{CONCLUSION}

Here, p(HEMA-co-EGDMA) hydrogel is reported on the application of antibiotic loaded hydrogel-based drug delivery system for a possible treatment of periodontitis. The drug release profile of $\mathrm{p}$ (HEMA) hydrogel has been investigated by using amoxicillin as the model drug. The structure, swelling characteristics and drug release performance of the amoxicillin loaded p(HEMA) hydrogel were evaluated in the study. The hydrogel disc sample has provided reswelling ability successfully. The swelling ratio has declined with the increasing amount of cross-linker. Higher amount of cross-linker caused increased hydrophobicity, smaller pore size and therefore the diffusion of solution has become more difficult. The drug release profile of the hydrogels was obtained from samples loaded in sink conditions and was consistent with the swelling results of hydrogels. The release rate of amoxicillin from H1 hydrogel was faster than $\mathrm{H} 2$ and $\mathrm{H} 3$ hydrogel showed the lowest release amount. As a result, the H1 hydrogel sample is a suitable material for drug delivery or biomedical application and can be assessed for further drug delivery studies.

\section{CONFLICTS OF INTEREST}

No conflict of interest was declared by the author.

\section{ACKNOWLEDGMENTS}

The author is grateful to Prof. Dr. Nihat TINKILIÇ for providing laboratory support.

\section{REFERENCES}

[1] Ayhan, H., Ayhan, F., "Water based PHEMA hydrogels for controlled drug delivery", Turkish Journal of Biochemistry, 43: 228-239, (2018).

[2] Cheaburu, C. N., Karasulu, H. Y., Yilmaz, O., "Chapter 13 - Nanoscaled dispersed systems used in drug-delivery applications", C. Vasile (Ed.), Polymeric Nanomaterials in Nanotherapeutics, Elsevier, 437-468, (2019).

[3] Peppas, A., Van Blarcom, S., "Hydrogel-based biosensors and sensing devices for drug delivery", Journal of Control Release, 240: 142-150, (2016).

[4] Balamurugan, D., Murali, M., Venkatesan, A., Nagasamy, V. D., "Hydrogel systems: Their classification, preparation and biomedical applications", International Journal of Pharmaceutical Research, 12: 180-187, (2020).

[5] Wei, W., Li, J., Qi, X., Zhong, Y., Zuo, G., Pan, X., Dong, W., "Synthesis and characterization of a multi-sensitive polysaccharide hydrogel for drug delivery", Carbohydrate Polymers, 177: 275283, (2017).

[6] George, J., Hsu, C. C., Nguyen, Ye, H., Cui, Z., "Neural tissue engineering with structured hydrogels in CNS models and therapies", Biotechnology Advances, 42: 107370, (2020). 
[7] Navaei, A., Saini, H., Christenson, W., Sullivan, R. T., Ros, R., Nikkhah, M., "Gold nanorodincorporated gelatin-based conductive hydrogels for engineering cardiac tissue constructs", Acta Biomaterialia, 41: 133-146, (2016).

[8] Kakuta, T., Takashima, Y., Nakahata, M., Otsubo, M., Yamaguchi, H., Harada, A., "Preorganized hydrogel: self-healing properties of supramolecular hydrogels formed by polymerization of hostguest-monomers that contain cyclodextrins and hydrophobic guest groups", Advanced Materials, 25: 2849-2853, (2013).

[9] Miyamae, K., Nakahata, M., Takashima, Y., Harada, A., "Self-healing, expansion-contraction and shape-memory properties of a preorganized supramolecular hydrogel through host-guest interactions", Angewandte Chemie, 127: 9112-9115, (2015).

[10] Oliveira, I. M., Gonçalves, C., Shin, M. E., Lee, S., Reis, R. L., Khang, G., Oliveira, J. M., "Enzymatically crosslinked tyramine-gellan gum hydrogels as drug delivery system for rheumatoid arthritis treatment", Drug Delivery and Translational Research: An Official Journal of the Controlled Release Society, 11(3): 1288-1300, (2020).

[11] Torres-Luna, C., Fan, X., Domszy, R., Hu, N., Wang, N. S., Yang, A., "Hydrogel-based ocular drug delivery systems for hydrophobic drugs", European Journal of Pharmaceutical Sciences, 154: 105503, (2020).

[12] Antipova, C., Parunova, Y., Vishnevskaya, M., Grigorev, T., Lukanina, K., Krasheninnikov, S., Gotovtsev, P., "Flexible electroconductive hydrogel for biosensors and biofuel cells application", Proceedings of the 12th International Conference on Developments in eSystems Engineering (DeSE), Kazan, 513-517, (2019).

[13] Guo, X., Li, Y., Li, Y., Ye, Z., Zhang, J., Zhu, T., Li, F., “An L012@PAni-PAAm hydrogel composite based-electrochemiluminescence biosensor for in situ detection of $\mathrm{H}_{2} \mathrm{O}_{2}$ released from cardiomyocytes", Electrochimica Acta, 354: 136763, (2020).

[14] Zavrel, V., Stol, M., "p(HEMA) composite as allografting material during therapy of periodontal disease: three case reports", Biomaterials, 14: 1109-1112, (1993).

[15] Buxadera-Palomero, J., Fricke, K., Reuter, S., Gil, F.J., Rodriguez, D., Canal, C., "One-Step liquid phase polymerization of HEMA by atmospheric-pressure plasma discharges for Ti dental implants", Applied Sciences, 11: 662, (2021).

[16] Duruk, G., Gürbüz, T., "The relationship between periodontal health and dental caries/restoration in children: A review of the literature", Journal of Dental Faculty of Ataturk University, 28(3): 421-428, (2018).

[17] Passos, M. F., Dias, D. R. C., Bastos, G. N. T., Jardini, A. L., Benatti, A. C. B., Dias, C. G., Maciel Filho, R., "pHEMA hydrogels", Journal of Thermal Analysis and Calorimetry, 125: 361-368, (2016).

[18] Andrade-Vivero, P., Fernandez-Gabriel, E., Alvarez-Lorenzo, C., Concheiro, A., "Improving the loading and release of NSAIDs from pHEMA hydrogels by copolymerization with functionalized monomers", Journal of Pharmaceutical Sciences, 96: 802-813, (2007).

[19] Hrioua, A., Loudiki, A., Farahi, A., Bakasse, M., Lahrich, S., Saqrane, S., El Mhammedi, M. A., "Recent advances in electrochemical sensors for amoxicillin detection in biological and environmental samples", Bioelectrochemistry, 137: 107687, (2021). 
[20] Fallahzadeh, R. A., Mahvi, A. H., Meybodi, M. N., Ghaneian, M. T., Dalvand, A., Salmani, M. H., Ehrampoush, M. H., "Application of photo-electro oxidation process for amoxicillin removal from aqueous solution: Modeling and toxicity evaluation", Korean Journal of Chemical Engineering, 36: 713-721, (2019).

[21] Iqbal, M. S., Akmal, M. A., "A simple method for simultaneous determination of some organic liquids in in-process materials and effluents produced in the manufacture of amoxicillin and ampicillin", Environmental Monitoring and Assessment, 151: 397-400, (2009).

[22] Morse, A., "Fate and effect of amoxicillin in space and terrestrial water reclamation systems", Phd. Thesis, Texas Tech University Institute of Science and Technology, Texas, 158-164, (2003).

[23] Johnson, A., Kong, F., Miao, S., Lin, H.T.V., Thomas S., Huang, Y.C., Kong, Z., "Therapeutic effects of antibiotics loaded cellulose nanofiber and $\kappa$-carrageenan oligosaccharide composite hydrogels for periodontitis treatment", Scientific Reports, 10: 18037, (2020).

[24] Di Benedetto, A., Gigante, I., Colucci, S., Grano, M., "Periodontal disease: Linking the primary inflammation to bone loss", Journal of Immunology Research, 503754: 1-7, (2013).

[25] Saini, R., Marawar, P. P., Shete, S., Saini, S., "Periodontitis, a true infection", Journal of Global Infectious Disease 1:149-150, (2009)

[26] Tariq, M., Iqbal, Z., Ali, J., Baboota, S., Talegaonkar S., Ahmad Z., Sahni, J., "Treatment modalities and evaluation models for periodontitis", International Journal of Pharmaceutical Investigation 2: 106-122, (2012)

[27] Sanjay, S. T., Zhou, W., Dou, M., Tavakoli, H., Ma, L., Xu, F., Li, X., "Recent advances of controlled drug delivery using microfluidic platforms", Advanced Drug Delivery Reviews, 128: 3-28, (2018).

[28] Nidhi, P., Anamika, C., Twinkle, S., Mehul, S., Hitesh, J., Umesh, U., “Controlled drug delivery system: A review”, Indo American Journal of Pharmaceutical Sciences, 3: 227-233, (2016).

[29] Joshi, D., Garg, T., Goyal, A. K., Rath, G., "Advanced drug delivery approaches against periodontitis", Drug Delivery, 23: 363-377, (2016).

[30] Suhail, M., Hsieh, Y.H., Shao, Y.F., Minhas, M.U., Wu, P.C., "Formulation and in-vitro characterization of $\mathrm{pH}$-responsive semi-interpenetrating polymer network hydrogels for controlled release of ketorolac tromethamine", Gels, 7: 167, (2021).

[31] Baliga, S., Muglikar, S., Kale, R., "Salivary pH: A diagnostic biomarker", Journal of Indian Society of Periodontology, 17: 461-465, (2013).

[32] Panda, N., Reddy A. V., Reddy, G. V. S., Panda, K., "Formulation design and in vitro evaluation of zolmitriptan immediate release tablets using primojel and AC-Di-Sol", Journal of Pharmaceutical Sciences and Research, 7: 545-553, (2015).

[33] Huang, C. W., Sun, Y. M., Huang, W. F., "Curing kinetics of the synthesis of poly(2-hydroxyethyl methacrylate) (PHEMA) with ethylene glycol dimethacrylate (EGDMA) as a crosslinking agent", Journal of Polymer Science Part A: Polymer Chemistry, 35: 1873-1889, (1997).

[34] Vargün, E., Usanmaz, A., "Degradation of poly(2-hydroxyethyl methacrylate) obtained by radiation in aqueous solution", Journal of Macromolecular Science, Part A: Pure and Applied Chemistry, 47: 882-891, (2010). 
[35] Yıldırım, E., "Synthesis and characterization of poly (2-hydroxyethyl methacrylate) homopolymer at room temperature via reversible addition-fragmentation chain transfer (RAFT) polymerization Technique" Gazi University Journal of Science, 33: 22-29, (2020).

[36] Dobic, S., Jovasevic, J., Vojisavljevic, M., Tomic, S., "Hemocompatibility and swelling studies of poly(2-hydroxyethyl methacrylate-co-itaconic acid-co-poly(ethylene glycol) dimethacrylate) hydrogels", Hemijska Industrija, 65: 675-685, (2011).

[37] Podkoscielna, B., Bartnicki, A., Gawdzik, B., "New crosslinked hydrogels derivatives of 2hydroxyethyl methacrylate: Synthesis, modifications and properties", Express Polymer Letters, 6: 759-771, (2012).

[38] Wei, W., Hu, X., Qi, X., Yu, H., Liu, Y., Li, J., Zhang, J., Dong, W., “A novel thermo-responsive hydrogel based on salecan and poly(N-isopropylacrylamide): Synthesis and characterization", Colloids and Surfaces B: Biointerfaces, 125: 1-11, (2015).

[39] Gibaldi, M., Feldman, S., "Establishment of sink conditions in dissolution rate determinations. Theoretical considerations and application to nondisintegrating dosage forms", Journal of Pharmaceutical Sciences, 56: 1238-1242, (1967).

[40] Huh, H. W., Zhao, L., Kim, S. Y., "Biomineralized biomimetic organic/inorganic hybrid hydrogels based on hyaluronic acid and poloxamer", Carbohydrate Polymers, 126: 130-140, (2015).

[41] Permanadewi, I., Kumoro, A. C., Wardhani, D. H., Aryanti, N., "Modelling of controlled drug release in gastrointestinal tract simulation", Journal of Physics: Conference Series, 1295: 012063, (2019). 